\title{
A Micromachined Photo-Supercapacitor Integrated with CdS-Sensitized Solar Cells and Buckypaper
}

\author{
Fu-Wei Lee, Cheng-Wen Ma, Yu-Hsuan Lin, \\ Po-Cheng Huang, Yen-Li Su, and Yao-Joe Yang* \\ Department of Mechanical Engineering, National Taiwan University, \\ No. 1, Sec. 4, Roosevelt Rd., Taipei 10617, Taiwan
}

(Received June 11, 2015; accepted December 11, 2015)

Keywords: buckypaper, micromachining technique, photo-supercapacitor, solar cell, $\mathrm{ZnO}$ nanorods

In this paper, the development of an all-solid-state photo-supercapacitor (PSC) by micromachining techniques is discussed. The device consists of two ZnO-nanorod solar cells, a buckypaper-based supercapacitor (SC), and an indium tin oxide-coated polyethylene terephthalate substrate. The two solar cells were connected in series by sputtered Au films for scaling up the output voltage. The proposed device is highly flexible, lightweight, and durable. The total photocharge voltage of the PSC exceeded 1.0 V. The energy conversion efficiency of the PSC was $0.26 \%$, and the maximum specific capacitance of the SC was $95.25 \mathrm{~F} / \mathrm{g}$. The photochargingdischarging process was measured. Cycle stability measurements showed that $95.89 \%$ of the initial specific capacitance was retained after 1000 cycles.

\section{Introduction}

To alleviate dependence on fossil fuels, many studies have focused on harvesting energy from renewable energy sources, such as wind, hydro, geothermal, and solar energies. Because solar energy is the cleanest and most abundant renewable energy source available, it is the most viable energy source for meeting energy demands and will become crucial in the future. Since the realization of silicon-based solar cells in the $1950 \mathrm{~s}$, numerous studies have developed low-cost and high-performance solar cells. ${ }^{(1-3)}$ Typical commercially available solar cells are stand-alone components that must be integrated with other discrete modules (e.g., batteries) for full operation. Such integration usually requires external electrical interconnection, leading to an extra energy loss. Therefore, miniaturized photo-supercapacitors (PSCs), which are realized by monolithically integrating solar cells with supercapacitors (SCs), offer promising energy storage applications, and have received considerable attention in recent years in solar energy industries. ${ }^{(4-6)}$

In general, according to device structures, PSCs can be categorized into a two-electrode type, ${ }^{(7,8)}$ a three-electrode type, ${ }^{(9)}$ or a core-shell wire type. ${ }^{(10)}$ The three-electrode configuration achieves a higher energy output than does the two-electrode system. Various three-electrode-type PSCs have been reported. Chen et al. ${ }^{(11)}$ presented a plastic PSC with a three-layer configuration. The proposed configuration separates a flexible solar cell and an SC by sharing a common platinum electrode. Wee et al. ${ }^{(12)}$ created a printable PSC by integrating an organic photovoltaic cell and a carbon-

"Corresponding author: e-mail: yjy@ntu.edu.tw 
nanotube-based SC. A PSC was demonstrated using an N3-dye-adsorbed $\mathrm{TiO}_{2}$ photoelectrode and PProDOT-Et2 polymer films as SC materials. ${ }^{(13)}$ Skunik-Nuckowska et al.(14) presented a solidstate PSC integrated with a dye-sensitized solar cell (DSSC) and an electrochemical capacitor. The device could operate under low light intensity conditions. Cohn et al. ${ }^{(15)}$ proposed a simple waferscale process in which a silicon wafer was used as the central electrode for the anode of a DSSC and an electrode for an electrochemical SC. Bae et al.(16) proposed hybrid devices for energy harvesting and storage along a microfiber using $\mathrm{ZnO}$ nanorods and graphene. Both mechanical and solar energies were simultaneously scavenged and stored in the devices. In addition, a microfiber that simultaneously achieves photoelectric conversion and energy storage was reported. ${ }^{(17)}$ The proposed device was realized by coaxially integrating a DSSC with an electrochemical capacitor.

In this work, we present a solid-state PSC with a three-electrode configuration by monolithically integrating a buckypaper-based SC with two CdS-sensitized solar cells (CdS-SSCs) and ZnO nanorods. Two CdS-SSCs were connected in series for scaling up the output voltage to increase the output power. The solar cells and SC were monolithically fabricated using micromachining techniques, thus reducing the energy loss between the solar cells and the SC. In addition, the device was realized on an indium tin oxide (ITO)-coated polyethylene terephthalate (PET) substrate, and polyvinyl alcohol-potassium hydroxide (PVA- $\mathrm{KOH}$ ) was used as the electrolyte. Therefore, the proposed PSC has advantages such as flexibility and simple device packaging. The measured cyclic voltammetry $(\mathrm{CV})$ curves and the photocharging and discharging curves of the device are provided. The rest of the paper is organized as follows: In $\S 2$, the device design and the device fabrication processes, including buckypaper preparation, synthesis of $\mathrm{ZnO}$ nanorod arrays, and fabrication of PSC, are presented. In $\S 3$, the measurement results are discussed, and finally, $\S 4$ contains our conclusions.

\section{Materials and Methods}

\subsection{Device design}

Figure 1 shows the design of the PSC, which includes two CdS-SSCs and an SC. The entire device was monolithically implemented in-plane on an ITO-coated PET sheet substrate. Interconnect Au was deposited and patterned on top of the device, and the two CdS-SSCs and SC

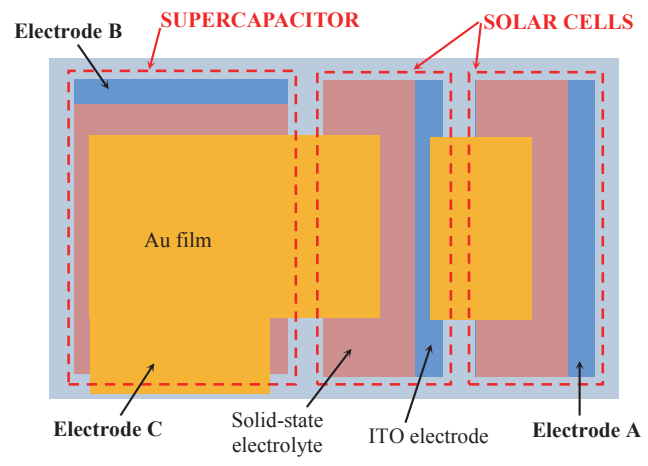

Fig. 1. (Color online) Device configuration of the photo-supercapacitor. 
were connected in series for scaling up the output voltage and directly storing the electricity in the SC with a three-electrode configuration. The CdS-SSCs are based on $\mathrm{ZnO}$ nanorods and CdS nanoparticles. The oriented crystalline $\mathrm{ZnO}$ nanorods are the units for harvesting solar energy. In addition, the CdS nanoparticles serve as a sensitizer of the CdS-SSCs and are deposited on the ZnO nanorod array uniformly. The SC consists of buckypaper electrodes with PVA-KOH gel electrolyte for storing the energy generated by CdS-SSCs.

As shown in Fig. 1, three electrodes were implemented in the device, Electrode A, Electrode B, and Electrode C. When Electrode A and Electrode B are connected, the device is ready to store the energy in the SC. When sunlight radiates on the CdS-SSCs, solar-generated electrons are injected into the conduction band of $\mathrm{ZnO}$ nanorods from $\mathrm{CdS}$ molecules and are transported to the $\mathrm{SC}$; the generated holes accumulate at the Au electrode. When Electrode B and Electrode C are connected with a load impedance, the energy stored in the SC can be supplied to the load.

$\mathrm{ZnO}$ nanorods are relatively durable for solar cell applications. ${ }^{(18)}$ Also, tidy rods of $\mathrm{ZnO}$ are advantageous for regulating the movement of charge carriers. Each $\mathrm{ZnO}$ nanorod can be considered as an n-type semiconductor with high electron mobility because of single-crystalline morphology. In general, electron transport in a $\mathrm{ZnO}$ nanorod is about a few orders of magnitude faster than that of a polycrystalline material.

In addition, because buckypapers are films of paperlike entangled CNT assemblies held together by van der Waals forces, they exhibit excellent properties such as high electrical conductivity, high thermal conductivities, high flexibility, and high mechanical stability. ${ }^{(19)}$ Furthermore, buckypapers have high effective surface areas due to the highly entangled CNT configuration, which is especially advantageous for supercapacitor applications that require large charge capacity.

\subsection{Buckypaper preparation}

Buckypaper was fabricated by filtering a single-walled carbon nanotube (SWCNT) solution by vacuum filtration. The $0.01 \mathrm{wt} \% \mathrm{SWCNT}$ solution was completely mixed using an ultrasonic agitator for $2 \mathrm{~h}$ to reduce the bundling tendency of the SWCNTs before filtration. The SWCNTs used in this study were 10-20 nm in diameter and 1-2 $\mu \mathrm{m}$ in length (Golden Innovation Business, Taiwan). A nylon membrane filter $\left(\mathrm{MS}^{\circledR}\right)$ with a pore size of $0.45 \mu \mathrm{m}$ was used for vacuum filtration. After vacuum filtration, the membrane filter with an SWCNT film was dried at room temperature for $2 \mathrm{~h}$, and the film was subsequently removed. The SWCNT film was a thick freestanding membrane, which is also known as a buckypaper. ${ }^{(20)}$ In this study, the thickness of the buckypaper was approximately $70 \mu \mathrm{m}$.

\subsection{Synthesis of $\mathrm{ZnO}$ nanorod arrays}

$\mathrm{ZnO}$ nanorod arrays were synthesized using a chemical solution method ${ }^{(21)}$ that involved a simple two-step process. In the first step, $\mathrm{ZnO}$ seeds were spin-coated on the top surface of an ITOPET substrate to form a $\mathrm{ZnO}$ crystal seed film (i.e., a seed layer). The process details of growing the $\mathrm{ZnO}$ seed layer were described by Greene et al. ${ }^{(22)}$ In the second step, $\mathrm{ZnO}$ was hydrothermally grown by immersing the ITO-PET substrate upside down in a dish filled with $0.025 \mathrm{M}$ aqueous zinc nitrate hexahydrate, $0.005 \mathrm{M}$ polyethylenimine, and $0.80 \mathrm{M}$ ammonium hydroxide at $95{ }^{\circ} \mathrm{C}$. ${ }^{23)}$ The reaction continued for $3 \mathrm{~h}$. In this study, the length of the $\mathrm{ZnO}$ nanorod was approximately $10 \mu \mathrm{m}$. Figure 2 shows the scanning electron microscopy image of the $\mathrm{ZnO}$ nanorod arrays. 


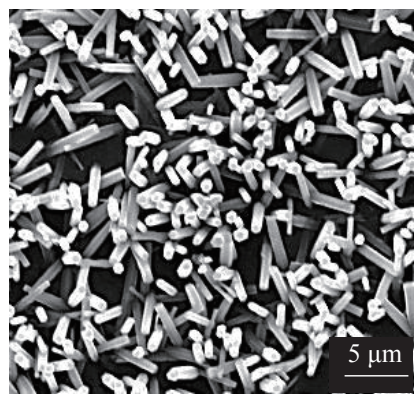

(a)

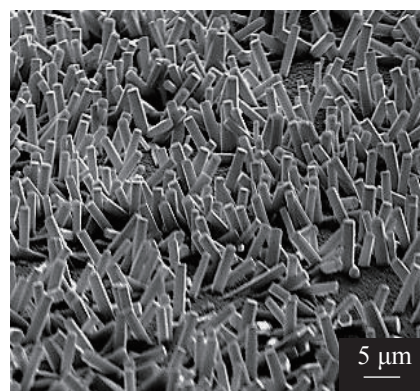

(b)

Fig. 2. SEM images of $\mathrm{ZnO}$ nanorod arrays: (a) top view and (b) side view.

\subsection{Fabrication of PSC}

The fabrication process is shown in Fig. 3. First, the ITO-PET substrate was patterned as three electrodes [Figs. 3(a) and 3(b)]. The texture $\mathrm{ZnO}$ seed layer was placed on the substrate and subsequently dried in air [Fig. 3(c)]. The coated substrate was annealed at $150{ }^{\circ} \mathrm{C}$ for $20 \mathrm{~min}$ to form $\mathrm{ZnO}$ nanoparticles (NPs), which were patterned using reactive-ion etching [Fig. 3(d)]. Subsequently, $\mathrm{ZnO}$ nanorods were grown, and a CdS sensitizer was coated [Fig. 3(e)]. A chemical bath deposition method was used for embedding CdS NPs at room temperature. ${ }^{(24)}$ The SC consisted of two SWCNT film electrodes with an identical composition separated by a PVA-KOH electrolyte [Fig. 3(f)]. The electrolyte was dispensed on the $\mathrm{ZnO}$ nanorod layer and buckypaper [Fig. (g)]. Finally, a Au film was deposited as an electrode and electrical connection layer [Fig. 3(h)]. The fabricated PSC is shown in Fig. 4. The size of each solar cell was approximately $5 \times 10 \mathrm{~mm}^{2}$, and the size of the SC was approximately $10 \times 10 \mathrm{~mm}^{2}$.

\section{Results and Discussion}

Figure 5 shows the current-voltage curves of the solar cell. Measurements were obtained using a source meter (Keithley 2400). Simulated AM $1.5 \mathrm{G}$ illumination $\left(100 \mathrm{~mW} / \mathrm{cm}^{2}\right)$ was provided using a solar light source (Newport class A), and the light intensity was measured using a calibrated Si solar cell. The total area of the photoanode for measurement was $0.5 \mathrm{~cm}^{2}$, and the voltage was scanned from -1 to $1 \mathrm{~V}$ with a fixed increment of $0.025 \mathrm{~V}$. In Fig. 5, the solar cell efficiency, fill factor, open-circuit voltage, and short-circuit current were $0.26 \%, 0.35,0.55 \mathrm{~V}$, and $2.0 \mathrm{mAcm}^{-2}$, respectively. The CV curves of the fabricated SC at various scan rates $(10-200 \mathrm{mV} / \mathrm{s})$ are shown in Fig. 6. The shape of the CV curves was mirrored with respect to the line of zero current. Furthermore, the CV curves showed a rapid response to current as the voltage scanning was reversed. This behavior indicates that the buckypaper SC possesses an electrical double-layer capacitance with small equivalent series resistance and has a high electrochemical reversibility. Typically, specific capacitance can be evaluated from the measured CV curves using the following equation: ${ }^{(25)}$

$$
C=\frac{A R E A_{\mathrm{CV}}}{m \cdot s \cdot \Delta V}
$$




\section{(a) ITO-PET film}

(b) Patterned ITO

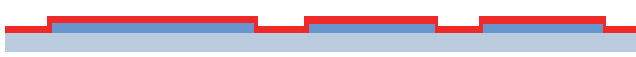

(c) Spin coating of $\mathrm{ZnO}$ seed layer

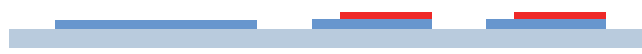

(d) RIE of ZnO NPs

(e) Growing $\mathrm{ZnO}$ nanorods and coated $\mathrm{CdS}$ sensitizer

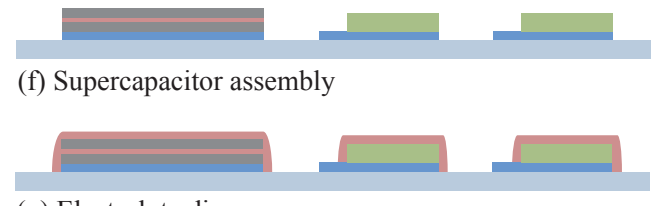

(g) Electrolyte dispenser

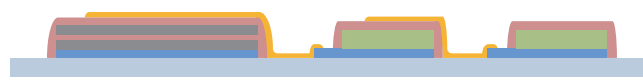

(h) Au film deposited

ITO

$\mathrm{ZnO} \mathrm{NPs}$

Electrolyte

Au film

PET $\quad$ ZnO Nrs + CdS

Buckypaper

Fig. 3. (Color online) Fabrication process for the PSC.

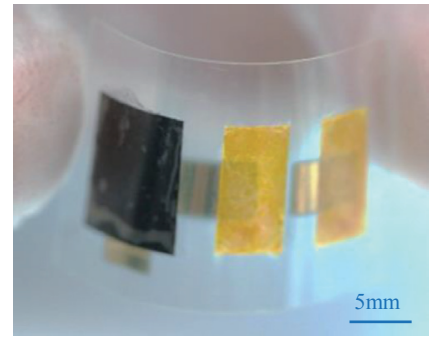

Fig. 4. (Color online) Fabricated PSC.

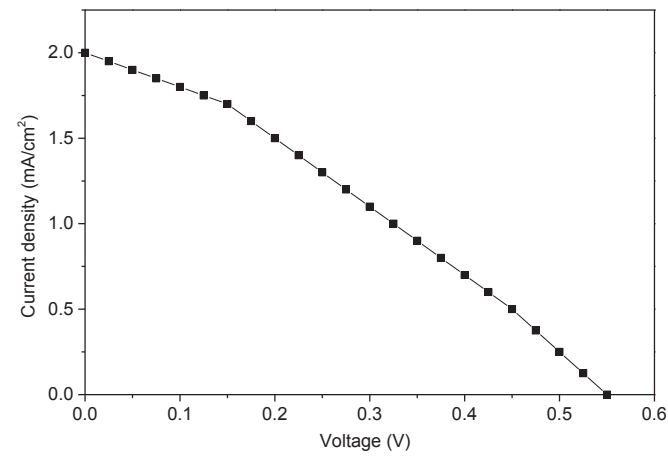

Fig. 5. Measured $I-V$ curve of solar cell.

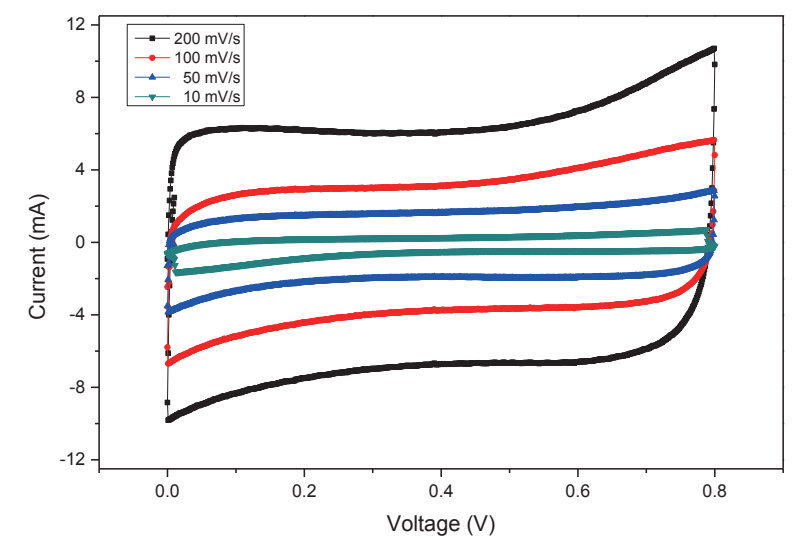

Fig. 6. (Color online) CV curves of SC at different scan rates.

where $A R E A_{\mathrm{CV}}$ is the area integral of the voltammogram (i.e., the total voltammetric charge obtained by integrating the positive and negative sweeps in a cyclic voltammogram), $s$ is the scan rate, $m$ is the mass of the electrode, and $\Delta V$ is the scanned potential window. The specific capacitance 
decreased from 95.25 to $62.5 \mathrm{~F} / \mathrm{g}$ when the potential scan rate increased from 10 to $200 \mathrm{mV} / \mathrm{s}$. For low scan rates, the $C$ values become higher because ions have sufficient time to penetrate and reside in almost all the available pores of the buckypaper electrodes and form electric double layers, which results in higher capacitance. In contrast, at a high scan rate, the ions might not have enough time to penetrate in the available pores of electrodes to form electric double layers, which in turn decreases the capacitance. ${ }^{(26)}$

Figure 7 shows the plot of the capacitance retention ratio versus the number of repeating CV cycles at a scan rate of $1 \mathrm{~V} / \mathrm{s}$. After 1000 cycles, $95.89 \%$ of the initial specific capacitance of the device was retained. The inset in the figure shows the CV curves of the first, 250th, 750th, and 1000th cycles. Figure 8(a) shows the plot of voltage versus the time required for photocharging and discharging. During charging, Electrode A and Electrode B were shorted [Fig. 8(b)], and the PSC was charged by illumination under $100 \mathrm{mWcm}^{-2}$ for $200 \mathrm{~s}$. The electrons photogenerated by the solar cells flowed to the SC and were stored in the buckypaper SC. The photovoltage formed on the solar cells was the maximum charging voltage of the SC. The photovoltage increased

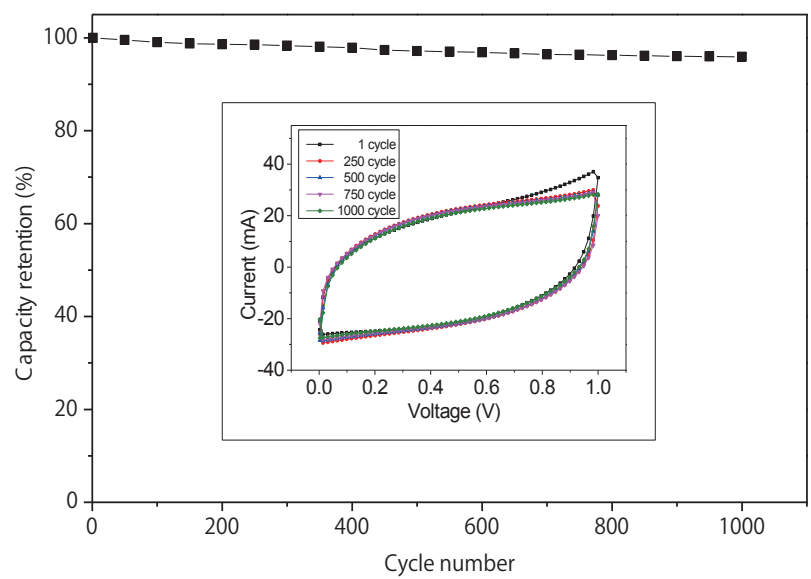

Fig. 7. (Color online) Cycle stability of micro-SC at a scan rate of $1 \mathrm{~V} / \mathrm{s}$. The inset shows the CV curves of the 1 st, 250th, 750th, and 1000th cycles.

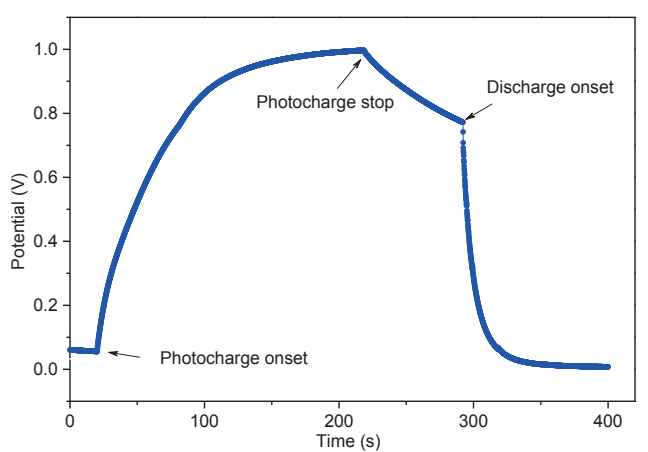

(a)

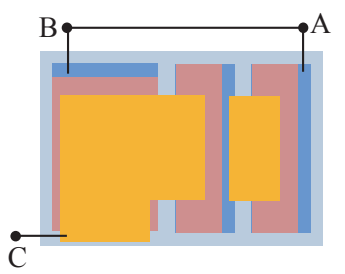

(b)

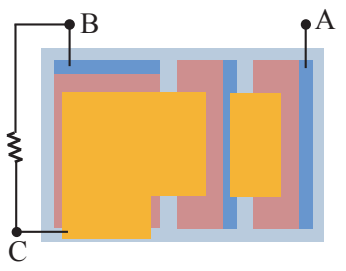

(c)

Fig. 8. (Color online) (a) Curves of voltage vs time of photocharging and discharging. (b) Configuration for photocharging. (c) Configuration for discharging. 
rapidly and reached a maximum of $1 \mathrm{~V}$ with $180 \mathrm{~s}$ of illumination. After charging for $200 \mathrm{~s}$, the illumination was stopped, and Electrode A and Electrode B were disconnected for $100 \mathrm{~s}$. The voltage of the SC decreased gradually, and a small drop was observed, indicating that the SC selfdischarged slowly within $100 \mathrm{~s}$. The SC was then discharged with a loading resistance [Fig. 8(c)]. The voltage rapidly decreased to $0 \mathrm{~V}$ as the stored energy dissipated through the resistor via Joule heating. For the proposed device, two CdS-SSCs were designed and connected in series so that the output voltage was scaled up. The experiment results showed that the total photocharge voltage of PSC was approximately $1.0 \mathrm{~V}$, which was higher than that shown in earlier work. ${ }^{(10,12,13)}$ Potentially, the proposed device is more suitable for applications of low-power integrated circuit devices.

\section{Conclusions}

In this study, we fabricated a solid-state PSC by integrating a buckypaper-based SC and two CdS-SSCs with ZnO nanorods. Featuring an ITO-coated PET substrate and PVA-KOH electrolyte, the device is flexible, and the packaging process for the device is simple. The experimental results of the PSC, including CV and photocharging-discharging behavior, were presented. The total photocharge voltage of PSC was approximately $1.0 \mathrm{~V}$. The energy conversion efficiency of the PSC was $0.26 \%$, and the maximum specific capacitance of SC was $95.25 \mathrm{~F} / \mathrm{g}$. Cycle stability measurements revealed that $95.89 \%$ of the initial specific capacitance was retained after 1000 cycles.

\section{Acknowledgements}

This study was supported in part by the National Science Council, Taiwan, R.O.C. (Contract No: NSC 100-2221-E-002-075-MY3).

\section{References}

1 M. A. Green, K. Emery, Y. Hishikawa, W. Warta, and E. D. Dunlop: Prog. Photovolt: Res. Appl. 23 (2015) 1.

2 J. You, L. Dou, K. Yoshimura, T. Kato, K. Ohya, T. Moriarty, K. Emery, C.-C. Chen, J. Gao, G. Li, and Y. Yang: Nat. Commun. 4 (2013) 1446.

3 H. Zhou, Q. Chen, G. Li, S. Luo, T.-B. Song, H.-S. Duan, Z. Hong, J. You, Y. Liu, and Y. Yang: Science 345 (2014) 542.

4 T. Miyasaka and T. N. Murakami: Appl. Phys. Lett. 85 (2004) 3932.

5 J. Xu, H. Wu, L. Lu, S.-F. Leung, D. Chen, X. Chen, Z. Fan, G. Shen, and D. Li: Adv. Funct. Mater. 24 (2014) 1840.

6 N. Bagheri, A. Aghaei, M. Y. Ghotbi, E. Marzbanrad, N. Vlachopoulos, L. Häggman, M. Wang, G. Boschloo, A. Hagfeldt, M. Skunik-Nuckowska, and P. J. Kulesza: Electrochim. Acta. 143 (2014) 390.

7 X. Huang, X. Zhang, and H. Jiang: J. Power Sources 248 (2014) 434.

8 X. Zhang, X. Huang, C. Li, and H. Jiang: Adv. Mater. 25 (2013) 4093.

9 T. N. Murakami, N. Kawashima, and T. Miyasaka: Chem. Commun. 14 (2005) 3346.

10 T. Song and B. Sun: ChemSusChem 6 (2013) 408.

11 H.-W. Chen, C.-Y. Hsu, J.-G. Chen, K.-M. Lee, C.-C. Wang, K.-C. Huang, and K.-C. Ho: J. Power Sources $195(2010) 6225$.

12 G. Wee, T. Salim, Y. M. Lam, S. G. Mhaisalkar, and M. Srinivasan: Energy Environ. Sci. 4 (2011) 413.

13 C.-Y. Hsu, H.-W. Chen, K.-M. Lee, C.-W. Hu, and K.-C. Ho: J. Power Sources 195 (2010) 6232.

14 M. Skunik-Nuckowska, K. Grzejszczyk, P. J. Kulesza, L. Yang, N. Vlachopoulos, L. Häggman, E. Johansson, and A. Hagfeldt: J. Power Sources 234 (2013) 91. 
15 A. P. Cohn, W. R. Erwin, K. Share, L. Oakes, A. S. Westover, R. E. Carter, R. Bardhan, and C. L. Pint: Nano Lett. 15 (2015) 2727.

16 J. Bae, Y. J. Park, M. Lee, S. N. Cha, Y. J. Choi, C. S. Lee, J. M. Kim, and Z. L. Wang: Adv. Mater. 23 (2011) 3446.

17 X. Chen, H. Sun, Z. Yang, G. Guan, Z. Zhang, L. Qiu, and H. Peng: J. Mater. Chem. A. 2 (2014) 1897.

18 Y. Hames, Z. Alpaslan, A. Kösemen, S. E. San, and Y. Yerli: Sol. Energy 84 (2010) 426.

19 J. Che, P. Chen, and M. B. Chan-Park: J. Mater. Chem. A 1 (2013) 4057.

20 M. Endo, H. Muramatsu, T. Hayashi, Y. A. Kim, M. Terrones, and M. S. Dresselhaus: Nature 433 (2005) 476.

21 L. Vayssieres, K. Keis, S.-E. Lindquist, and A. Hagfeldt: J. Phys. Chem. B 105 (2001) 3350.

22 L. E. Greene, M. Law, J. Goldberger, F. Kim, J. C. Johnson, Y. Zhang, R. J. Saykally, and P. Yang: Angew. Chem. Int. Ed. 42 (2003) 3031.

23 M. Law, L. E. Greene, J. C. Johnson, R. Saykally, and P. Yang: Nat. Mater. 4 (2005) 455.

24 R. S. Mane and C. D. Lokhande: Mater. Chem. Phys. 65 (2000) 1.

25 Y. Jin, H. Chen, M. Chen, N. Liu, and Q. Li: ACS Appl. Mat. Inter. 5 (2013) 3408.

26 R. Farma, M. Deraman, Awitdrus, I. A. Talib, R. Omar, J. G. Manjunatha, M. M. Ishak, N. H. Basri, and B. N. M. Dolah: Int. J. Electrochem. Sci. 8 (2013) 257. 